\title{
Binocular summation of
}

\section{equal-energy flashes of unequal duration*}

\section{H. WESTENDORF, R. RANDOLPH BLAKE, and ROBERT FOX \\ Vanderbilt University, Nashville, Tennessee 37240}

To determine if binocular summation occurs when increment flashes are of equal energy (Bloch's law) but unequal in luminance-duration parameters, three Ss made temporal forced-choice judgments: (1) monocularly, (2) binocularly when the flashes to each eye were identical, (3) binocularly when the flashes to each eye were of equal energy but different in terms of their luminance and duration parameters, and (4) binocularly when flashes to each eye were separated by $100 \mathrm{msec}$. Binocular detection rates were consistently superior to monocular detection rates. Similarity in performance between Conditions 2 and 3 indicates that the binocular visual system responds only to the total energy of each monocular flash. The data from two Ss reveal that binocular performance was greater than that predicted on the basis of probability summation.

The extent to which two eyes facilitate performance relative to a single eye has been a persistent issue in vision research. Research on this issue is generally catalogued under the rubric of binocular summation. Several recent investigations have revealed that binocular performance is superior for several kinds of threshold situations, including detection of incremental light flashes (e.g., Collier, 1954; Matin, 1962), recognition of letter forms (e.g., Eriksen, Greenspon, Lappin, \& Carlson, 1966), and Landolt ring acuity (e.g., Kahnemann, Norman, \& Kubovy, 1967), Moreover, since the magnitude of the difference between binocular and monocular performance has been greater than that predicted on the basis of probability summation, it is necessary to conclude that the superiority of binocular performance is due to some form of facilitatory neural interaction between the two eyes.

Virtually all experiments on binocular summation have used situations in which the monocular stimuli are identical to each other in every respect. It would be interesting to know whether these conditions are critical for demonstrating the superiority of binocular vision or whether summation is a more robust phenomenon that would occur when stimuli differed. The purpose of the present experiment is to determine if binocular summation occurs in an increment detection task when the monocular test flashes are equal in energy but different in terms of their luminance and duration parameters. That is, the flashes are of equal energy in accord with Bloch's law, but one

*This research was supported in part by Grant EY 00590 from the Eye Institute, NIH. We thank Joseph Lappin for comments on an earlier version of the paper. flash is of longer duration and lower luminance, while its partner is of shorter duration but higher luminance. The detectability of these flashes, unequal in terms of their luminance-duration configurations, was compared to that of equal flashes. If the binocular detection system responds only to total energy, then both equal and unequal flashes would be equally detectable. But if the system is sensitive to the difference in duration and luminance between the flashes, it may regard them as independent or even inhibitory, in which case the binocular detectability of unequal flashes would be less than the detectability of equal flashes.

\section{METHOD}

Subjects

Three Ss were paid to participate in the experiment. R.B. was a highly experienced psychophysical observer and had excellent uncorrected vision. B.W. was highly experienced and had vision corrected to normal via contact lenses. J.N. was an unexperienced observer with excellent uncorrected vision.

Apparatus
The basic apparatus was a custom-built haploscope constructed of heavy aluminum stock and rigidly mounted on a steel bench. The left-eye and right-eye field arms were $1 \mathrm{~m}$ long; each of the arms had an auxiliary arm attached at a right angle to it. The eye-field arms pivoted at the viewing port under the pivot points of the eyes in such a way that both interpupillary distance and convergence angle were independently adjustable. The haploscope and related apparatus were enclosed in a lightproof housing fitted with internal baffles to prevent interference from stray light. An adjustable biteboard and headrest were located at the viewing port of the housing. Also at the viewing port, a pair of trial frames was suspended from an assembly that permitted fine adjustment of the frames in three dimensions. The trial frames carried artificial pupils whose 2-mm aperture was located in a protruding conical portion so that it could be located as close as possible to the cornea.

The background displays on which the test flashes were superimposed were mounted towards the ends of both arms of the haploscope, $75 \mathrm{~cm}$ from each eye. These displays were made from high-contrast negatives (Kodalith) mounted on translucent Plexiglas sheets and transilluminated by $15-W$ incandescent bulbs powered by a well-regulated dc power supply. The configuration of the displays as seen by the $S$ is illustrated in Fig. 1. A solid white 1-deg disk, which formed the center of the display, was surrounded by a 7-deg 30-min black annulus that was surrounded, in turn, by a 30-min-wide white ring. These concentric contours served to maintain fusion. To provide a fixation point and to define the area in which test flashes would appear, black threads were used to form diagonal crosshairs on the central 1-deg white disks. The adjustment of luminance of these displays was made with neutral density filters interposed between the incandescent bulb and the Plexiglas sheet. The luminance of the white portion of the background display was $10.2 \mathrm{~cd} / \mathrm{m}^{2}$; the luminance of the black or opaque portions of the display was less than $.005 \mathrm{~cd} / \mathrm{m}^{2}$. The luminance levels were continuously monitored throughout the experimental session via photocells.

Test flashes were produced by cold-cathode mercury-argon lamps placed in lamp houses mounted on the auxiliary arms of the haploscope. The lamps were powered and their duration controlled by a lamp-driver unit and timing units of a tachistoscope (Scientific Prototype Model GA). A collimating lens, neutral filters, and masks located within the lamp house produced 7-min circular homogeneously illuminated flashes. By means of beam splitters, the test flashes were optically superimposed on the right quadrant of the 1 -deg disk. The center of the test flash was located $15 \mathrm{~min}$ from the intersection of the fixation crosshairs. The luminance of the test flash was varied by changing neutral density filters in the lamp house. Two luminance values were used for all $S_{8}$ for all conditions; the flash of lower luminance was $3.4 \mathrm{~cd} / \mathrm{m}^{2}$, and the flash of higher luminance $\mathrm{was} 10.2 \mathrm{~cd} / \mathrm{m}^{2}$.

Absolute luminance values were measured before each experimental session with a Pritchard Spectra spot 


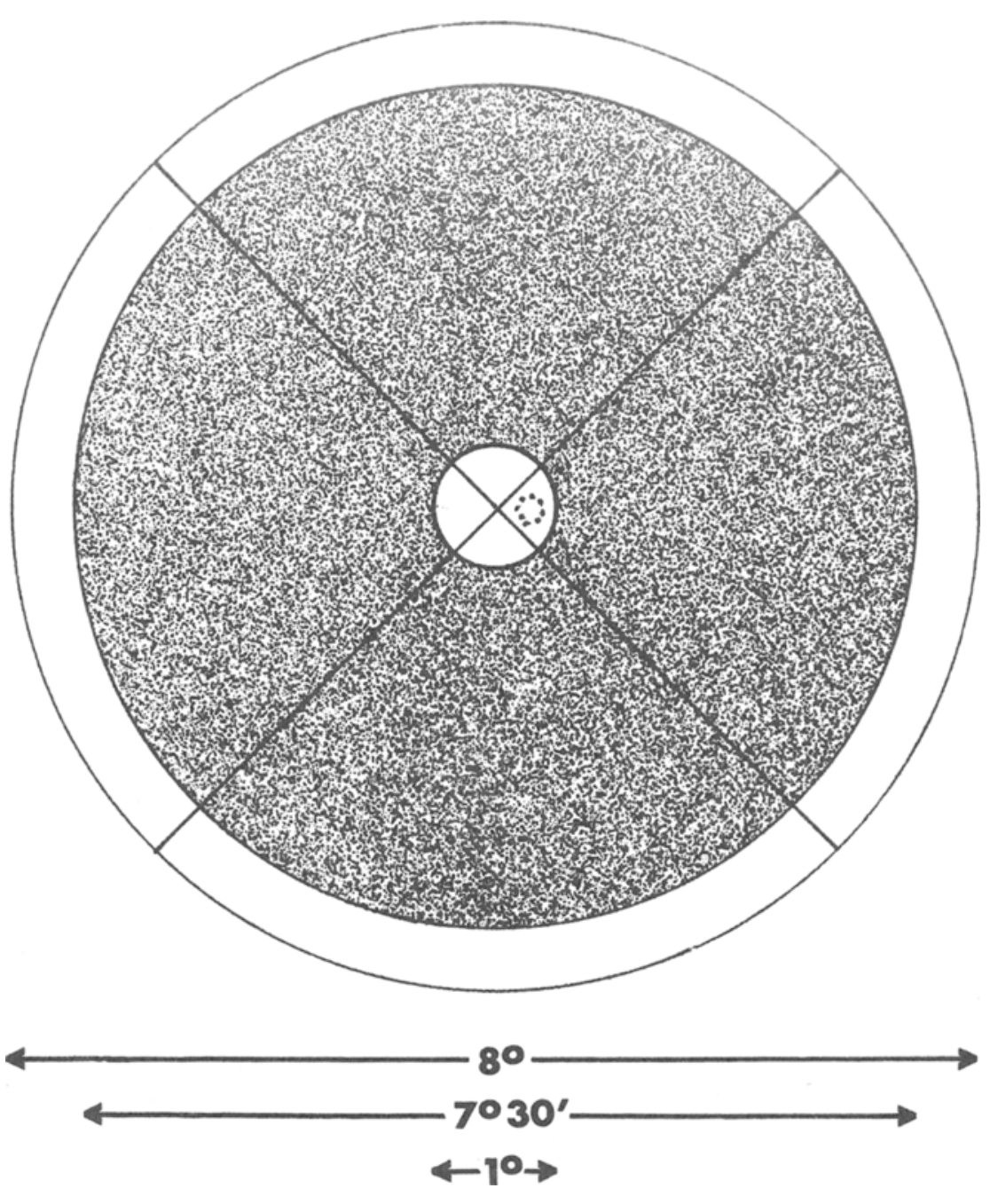

Fig. 1. Stimulus configuration. The small broken circle indicates the position of the test flash.

photometer (Model 1970-PR). The waveform of the test flashes was examined using a phototube and oscilloscope. For both the left-eye lamp and right-eye lamp, the shape of the wave was square, with the rise to maximum amplitude occurring in less than $1 \mathrm{msec}$, the rise to $90 \%$ of maximum amplitude occurring within .5 msec; decay to zero amplitude was complete within .5 msec.

To mask various extraneous sounds, a moderate level of white noise was presented to the $s$ through headphones. To aid in securing a comfortable position while constrained by the biteboard, the $S$ sat in a motorized seat (an automobile power seat) that could be adjusted in several dimensions.

\section{Procedure}

Each trial throughout the experiment constituted a three-choice temporal forced-choice task with levels. The results of these sessions were used to establish two durations (differing by a factor of 3 ) for each $\mathrm{S}$ to be used in the experimental sessions. The long durations for $\mathrm{Ss}$ R.B., B.W., and J.N. were 15, 24, and $18 \mathrm{msec}$, respectively; the short durations were one-third of these respective values.

Increment detection thresholds were measured under four conditions: (1) a monocular condition in which flashes were presented to each eye alone; (2) a binocular identical-flash condition in which the right-eye and left-eye test flashes were of identical luminance and duration and were presented simultaneously; (3) a binocular unequal-flash condition in which the flashes were of unequal luminance and duration and were presented simultaneously (with onsets synchronous); and (4) a binocular asynchronous condition in which identical flashes were presented separated by $100 \mathrm{msec}$. This last condition was included in order to provide an empirical baseline for assessment of probability summation. It is known from other work (e.g., Matin, 1962) that flashes separated by this temporal interval do not interact.

From each $S, 320$ judgments for each of these four conditions were obtained in eight sessions. A session consisted of 16 randomized blocks of 10 trials each, with 4 blocks dedicated to each condition. Within each block, the experimental conditions were not varied. For all conditions, when appropriate, type of test flash and eye of origin were balanced across blocks.

The Ss were not informed as to the condition being tested in any given block. Each trial took about $15 \mathrm{sec}$, and short rest periods were given after each block. Under both monocular and binocular conditions, Ss kept both eyes open and maintained fusion of the background displays. Special attention was paid to ensuring that Ss achieved and maintained good fusion throughout a block. Ss were frequently reminded to fixate the crosshair intersection and never to initiate a trial if fusion was not present. Prior to the first trial of each session, Ss spent at least $10 \mathrm{~min}$ viewing the stimulus field, during which time fusional adjustments were made, alignment of the test probes was checked, and several practice trials were administered.

\section{RESULTS AND DISCUSSION}

The results of the experiment are summarized in Table 1 in terms of the percentage of correct detections for each $S$ under each of the four conditions. The two leftmost columns of Table 1 show the percentage of correct detections from the left-eye 
and right-eye monocular conditions. There is virtually no difference between the recognition scores for the two types of flashes, so the table values combine both brief flash and long flash detection scores. An examination of detection scores under all conditions across sessions revealed no systematic trends, indicating that Ss were performing at an asymptotic level throughout the experiment.

For all Ss, it is clear that the binocular detection scores under all three binocular conditions are higher than the monocular detection scores. Of particular interest is the comparison between the binocular identical-flash condition and the binocular unequal-flash condition. The similarity between these scores indicates that the binocular detection system does not distinguish between luminance and temporal characteristics of the flashes, but rather responds to the total energy in the flash.

Performance under the binocular asynchronous condition is useful in deciding whether or not the improvement in binocular performance is due to probability summation or to neural interaction. For Ss R.B. and B.W., the detection scores for both the binocular identical-flash condition and the binocular unequal-flash condition are greater than for the binocular asynchronous condition. On the assumption that the improvement in the binocular asynchronous condition over the monocular condition can be due only to probability summation, it is necessary to conclude that the superior performance exhibited by these two Ss under both binocular conditions with synchronous flashes must be due to neural interaction. On the other hand, the results of $S$ J.N. reveal no evidence for neural interaction. There is essentially no difference among his detection scores for any of the three binocular conditions. To test the hypothesis than J.N.'s performance under the binocular asynchronous condition could be attributable, at least in part, to neural summation extending across the 100-msec gap between flashes, a supplemental experiment was run on J.N., using a $200-\mathrm{msec}$ delay condition in addition to the 100-msec delay. Performance between these two conditions did not differ and was of the same magnitude as that observed in the main experiment. So it would appear that J.N.'s performance under the binocular asynchronous condition is due only to probability summation. There is some tendency for J.N. to perform at a higher detection level under the binocular identical-flash condition and binocular unequal-flash condition, but this is not significant.

Table 1

Percentage of Correct Detections for Each $S$ Under Each Experimental Condition and Probability-Summation Predictions of Two Probability Models

\begin{tabular}{|c|c|c|c|c|c|c|c|}
\hline \multirow[b]{2}{*}{$\mathbf{s}$} & \multicolumn{2}{|c|}{ Monocular } & \multicolumn{3}{|c|}{ Binocular } & \multicolumn{2}{|c|}{ Model Predictions } \\
\hline & Right & Left & $\begin{array}{l}\text { Identi- } \\
\text { cal }\end{array}$ & Unequal & $\begin{array}{l}\text { Asynchro- } \\
\text { nous }\end{array}$ & $\begin{array}{l}\text { Two- } \\
\text { State }\end{array}$ & $\begin{array}{c}\text { Integra- } \\
\text { tion }\end{array}$ \\
\hline RB & 55 & 54 & 80 & 83 & 64 & 69 & 64 \\
\hline BW & 56 & 57 & 80 & 80 & 68 & 72 & 66 \\
\hline JN & 64 & 49 & 77 & 76 & 73 & 73 & 67 \\
\hline
\end{tabular}

Perhaps J.N. is one of those persons who apparently have normal binocular visual systems yet exhibit relatively little binocular summation. Careful inspection of the published data of binocular summation experiments (Blake \& Fox, unpublished review) reveals that there are large individual differences in the magnitude of the differences between binocular and monocular performance. These data suggest that there may be individuals who show only small amounts of summation, even when care has been taken to eliminate the operation of peripheral variables that would depress binocular performance. If it is at all tenable to presume that there are persons who have visual systems that are organized to yield only minimal summation, then J.N.'s results may be accounted for by classifying him as a minimal summator. 1

In addition to providing an empirical baseline for evaluating performance observed under the two binocular synchronous conditions, the binocular asynchronous condition can also be used to appraise different models for estimating probability summation. Two recent models particularly appropriate for estimating probability summation when Ss are encouraged to guess, as in the forced-choice method, are those described by Eriksen (Eriksen, 1966; Eriksen, Greenspon, Lappin, \& Carlson, 1966) and by Green and Swets (1966). The column labeled "two-state model" in Table 1 contains predictions derived from a particular version of the Eriksen model described in Eriksen et al. ${ }^{2}$ The column labeled "integration model" contains predictions based on the theory of signal detection as described in Green and Swets. ${ }^{3}$ The predictions of both models agree reasonably well with one another and with the results obtained under the binocular asynchronous condition.

It should be noted that probability summation predictions based on the classic probability theorem for independence, in which the predicted value is obtained by adding the separate probabilities and subtracting their product, are not appropriate for the present data. Eriksen (1966) has pointed out that application of the classic theorem makes no allowance for the guessing component associated with each monocular hit rate, thereby generating inflated estimates of binocular performance.

The main conclusion supported by the results of the present experiment is that binocular summation in excess of probability summation can be obtained when the monocular flashes are of equal energy but unequal in terms of their luminance-duration characteristics. Presumably, the binocular detection system ignores these characteristics and acts on total energy in the stimulus. This conclusion, of course, must be tempered by the realization that the full range of luminance and duration values over which Bloch's law is generally believed to hold has not been sampled, and it is passible that for more extreme values summation would fail.

\section{REFERENCES}

COLLIER, G. Probability of response and intertrial association as functions of monocular and binocular stimulation. Journal of Experimental Psychology, $1954,47,75-83$.

ERIKSEN, C. W. Independence of successive inputs and uncorrelated error in visual form perception. Journal of Experimental Psychology, 1966, 72, 26-35.

ERIKSEN, C. W., GREENSPON, $T$, S., LAPPIN, J., \& CARLSON, W. A. Binocular summation in the perception of form at brief durations. Perception \& Psy chophysics, 1966, 1, 415-419.

GREEN, D. M., \& SWETS, J. A. Signal detection theory and psychophysics. New York: Wiley, 1966.

KAHNEMANN, D., NORMAN, J., \& KUBOVY, M. Critical duration for the resolution of form: Centrally or peripherally determined? Journal of Experimental Psychology, 1967, 73. 323-327.

MATIN, L. Binocular summation at the absolute threshold for peripheral vision. Joumal of the Optical Society of America, 1962, 52, 1276-1286.

SWETS, J. A. Signal detection and recognition by human observers. New York: Wiley, 1964 .

\section{NOTES}

1. Could $I . N$ 's performance be related to stereopsis? Prior to the experiment, all Ss displayed adequate stereoscopic ability on the Modified Orthoraterslide (Fry-Sheppard scale). Following a sugzestion by Dr. Herschel Leibowitz, J.N.'s stereopsis was examined more intensively after the experiment, using a graduated series of Julesz random-element stereograms and the Verhoff Stereopter. Both measures indicate that J.N. possesses excellent stereopsis.

2. The two-state model assumes that on any single trial $S$ is in one of two states: 
(1) the correct perception state, with associate hit rate of 1.0, or (2) the guessing state, with hit rate equal to the a priori probability of a correct guess. As Eriksen (1966) has argued, this model tends to overpredict performance expected from multiple independent chances to perceive, since in practice Ss have no trouble distinguishing at least three different subjective states about their judgment of stimulus presentation. These different stimulus, which are indicated by confidence ratings, seem to reflect different states of perceptual sensitivity since accuracy of performance covaries with the confidence idence ratings were not obtained in the present experiment, it was necessary to employ the more conservative two-state model.

3. Values of $d^{\prime}$ for a three-choice forced-choice task were estimated using the procedure described by Ehliot (in Swets, 1964).

(Received for publication May 12, 1972; revision received July 17, 1972.) 